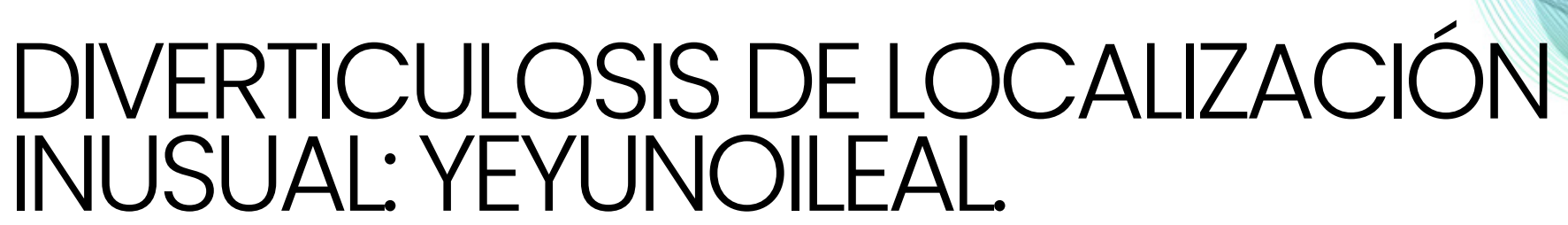

\title{
DIVERTICULUM OF UNUSUAL LOCATION: JEJUNOILEAL.
}

\section{Díaz Alcázar MM, Martín-Lagos Maldonado A, Ruiz Rodríguez AJ}

Hospital Universitario Clínico San Cecilio. Granada.

\section{Resumen}

La diverticulosis yeyunoileal es rara. Se estima que su prevalencia en pruebas de imagen es menor del $2 \%$.

Mujer de 63 años que consulta por dolor en hemiabdomen inferior y hasta 10-15 deposiciones diarias. En tomografía computarizada abdominal se objetivan hallazgos compatibles con diverticulitis aguda de íleon no complicada.

La diverticulosis yeyunoileal es mucho más infrecuente que en colon y duodeno. La diverticulitis es su complicación más frecuente. Es más habitual en edad avanzada, fundamentalmente a partir de la década de los 60 años. Es asintomática o produce sintomatología inespecífica. El diagnóstico es difícil y precisa de pruebas de imagen, por lo que probablemente esté infradiagnosticada. Es infrecuente que las complicaciones sean graves o recurrentes.

Palabras clave: diverticulosis, diverticulitis, yeyunoileal.

\section{CORRESPONDENCIA}

María del Mar Díaz Alcázar

Hospital Universitario Clínico San Cecilio

18016 Granada

mmardiazalcazar@gmail.com

Fecha de envio:18/02/2020

Fecha de aceptación: 29/02/2020

\section{Abstract}

Jejunoileal diverticulosis is an uncommon entity. Its prevalence on imaging tests is estimated to be less than $2 \%$.

A 63-year-old woman who consults for pain in the lower hemiabdomen and up to 10-15 daily stools. Abdominal computed tomography shows uncomplicated acute diverticulitis of the ileum.

Jejunoileal diverticulosis is much less common than at the colon and duodenum. Diverticulitis is the most common complication. It is more frequent in older patients, mainly over 60 years of age. It is asymptomatic or produces non-specific symptoms. The diagnosis is difficult and relies on imaging tests, so it is probably under-diagnosed. Complications are rarely severe or recurrent.

Keywors: diverticulosis, diverticulitis, jejunoileal.

\section{Cuerpo}

La diverticulosis yeyunoileal es rara. Se estima que su prevalencia en pruebas de imagen es menor del $2 \%{ }^{1,2}$.

Mujer de 63 años que consulta por dolor en hemiabdomen inferior y hasta 10-15 deposiciones diarias sin productos patológicos de pocos días de evolución. Analíticamente destaca elevación de reactantes de fase aguda. Se realiza tomografía computarizada abdominal que 
objetiva íleon redundante con divertículos y engrosamiento parietal difuso, así como hipercaptación parietal y estriación de la grasa circundante, con pequeña lengüeta de líquido libre y adenopatías locorregionales reactivas, todo ello en relación con diverticulitis aguda no complicada (Figuras 1 y 2). Buena evolución clínica con antibioterapia empírica.

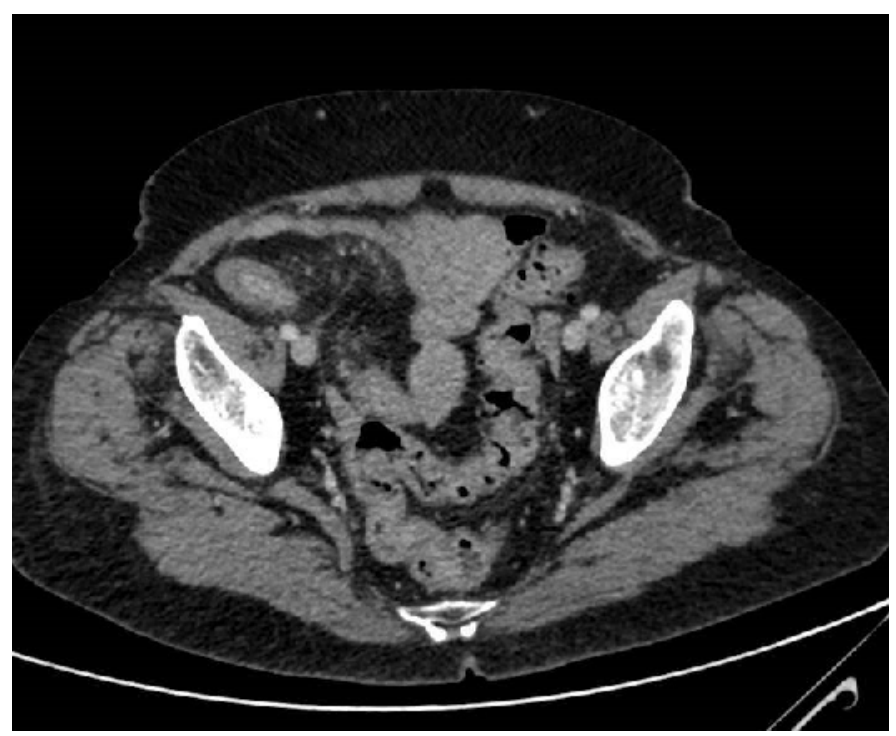

Figura 1

Corte transversal de tomografía computarizada abdominal en que se objetivan divertículos en íleon y colon.

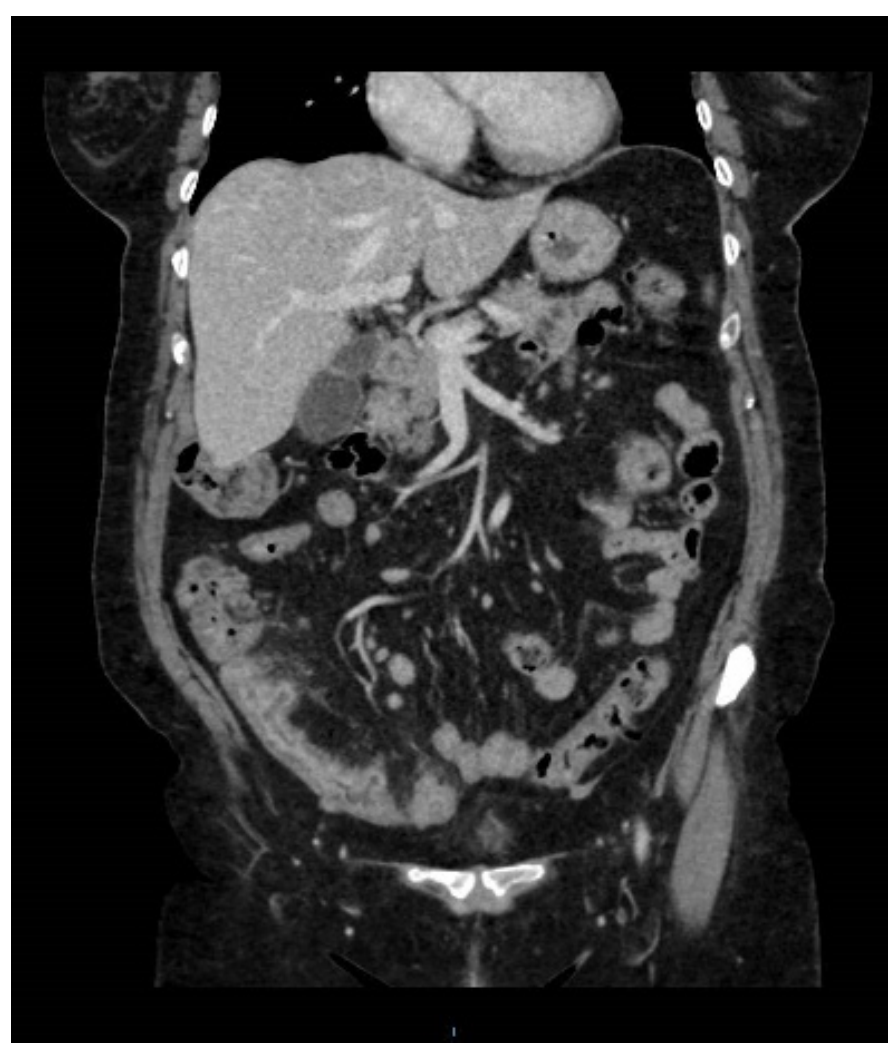

\section{Figura 2}

Corte coronal de tomografía computarizada abdominal en que se visualiza engrosamiento parietal difuso de íleon y divertículos a ese nivel y en colon.
La diverticulosis yeyunoileal es un hallazgo infrecuente, mucho más rara que en colon y duodeno ${ }^{1,2}$. Coexiste en el $30-75 \%$ de los pacientes con diverticulosis colónica ${ }^{3}$. La diverticulitis yeyunoileal, como en el caso presentado, es su complicación más frecuente, sin considerar el divertículo de Meckel, y puede aparecer en hasta el $6 \%$ de los $\operatorname{casos}^{1,2}$. Habitualmente los divertículos se desarrollan en el borde mesentérico del intestino delgado y son múltiples ${ }^{3}$. Son más habituales en yeyuno proximal, y la probabilidad de encontrarlos disminuye cerca de la válvula ileocecal ${ }^{3}$. Suelen desarrollarse en individuos de edad avanzada, fundamentalmente a partir de la década de los 60 años $^{1-3}$. La fisiopatología de la diverticulosis yeyunoileal es desconocida $^{2}$. Las hipótesis se centran en alteraciones del músculo liso o el plexo mientérico, discinesia intestinal o aumento de la presión intraluminal $^{2}$. Es asintomática o produce sintomatología inespecífica, salvo complicación como diverticulitis, sangrado o perforación ${ }^{1-3}$. El diagnóstico es difícil y precisa de pruebas de imagen, por lo que probablemente esté infradiagnosticada ${ }^{1,2}$. No hay consenso sobre el tratamiento, en parte por su baja frecuencia, aunque es raro que se precise cirugía ${ }^{1-3}$. Es infrecuente que las complicaciones derivadas de la diverticulosis yeyunoileal sean graves o recurrentes ${ }^{1,3}$.

\section{Bibliografía}

1. Lebert P, Millet I, Ernst O, Boulay-Coletta I, Corno L, Taourel P et al. Acute jejunoileal diverticulitis: multicenter descriptive study of 33 patients. AJR Am J Roentgenol 2018;210(6):1245-1251.

2. Lebert $P$, Ernst $O$, Zins $M$. Acquired diverticular disease of the jejunum and ileum: imaging features and pitfalls. Abdom Radiol 2019;44:1734-1743.

3. Ramistella AM, Brenna M, Fasolini F, De Monti M. Jejuno-ileal diverticulitis: A disorder not to underestimate. Int J Surg Case Rep 2019;58:81-84. 